\title{
Experimental Study on Watermark Interference in Multiple Re-Watermarking
}

\author{
Daniel Mark ${ }^{1}$, Andreas Uhl ${ }^{1,2}$, and Hartmut Wernisch ${ }^{2}$ \\ ${ }^{1}$ School of Telematics \& Network Engineering, Carinthia Tech Institute (CTI), Austria \\ ${ }^{2}$ Department of Computer Sciences, Salzburg University, Austria
}

\begin{abstract}
Watermark interference is a threat to reliable detection in multiple re-watermarking scenarios. The impact of using disjoint frequency bands and/or different embedding domains in limiting those interferences is evaluated and compared. Employing disjoint frequency bands for embedding different watermarks turns out to be more effective and is capable of maintaining reasonable detection correlation in multiple embedding applications.
\end{abstract}

Keywords: multiple watermarking, re-watermarking, robust watermarks

\section{INTRODUCTION}

Watermarking ${ }^{1,2}$ has been proposed as a generic technique to solve various problems associated with topcis in the areas of digital rights management (DRM) and multimedia security. ${ }^{3}$ Among other applications, watermarks can be used to control and monitor actions performed on the cover medium (e.g. "copy control watermarks"), to convey ownership information ("copyright watermarks"), to verify that object content has not changed ("authentication watermarks"), and to provide object-specific informations or captions ("annotation watermarks"). According to the respective applications, watermarking technology exhibits significantly different properties, e.g. with respect to robustness (as required for ownership claims) or fragility (as required for integrity investigations). Whereas watermarking has evolved to a mature technology in the last decade, several issues remain to be solved until large scale deployment is to be expected. Multiple watermarking is one of those issues.

Mintzer et al. ${ }^{4}$ discuss the latter three abovementioned types of watermarking applications in the context of multiple watermarking and identify different ways how to employ and to interpret multiple watermarking. Multiple watermarks can be used to address multiple applications or one application may be addressed several times. For example, a first watermark can be used to embed ownership information, a second one for integrity verification, and a third one for captioning. On the other hand, there can be multiple copyright watermarks, multiple verification watermarks, or multiple watermarks for multiple captions.

Focussing on the way how single watermarking techniques are actually fused into multiple watermarking schemes, Sheppard et al. ${ }^{5}$ distinguish three main categories of multiple watermarking techniques:

1. Composite watermarking: All watermarks are combined into a single watermark which is subsequently embedded in one single embedding step.

2. Segmented watermarking: The host data is partitioned into disjoint segments and each watermark is embedded into its specific share.

3. Successive watermarking: Watermarks are embedded one after the other. This approach is also denoted Rewatermarking in literature. Interactive embedding is a variant where the distortions imposed by successive embedding are examined and an embedding step is rolled back and re-applied with different parameters in case of too large degradation.

E-mail: uhl@cosy.sbg.ac.at 
In this work, we focus on multiple re-watermarking using robust embedding techniques. An overview of multiple watermarking technology as described in literature is given in Section 2. In Section 3 we will identify the technological requirements for our target application scenario based on the results of Section 2 and we will discuss multiple re-watermarking schemes with emphasis on the interference of the watermarks embedded. Corresponding re-watermarking experiments are described in Section 4 and Section 5 concludes the paper.

\section{MULTIPLE WATERMARKING}

Apart from Sheppard et al. ${ }^{5}$ composite and successive watermarking are hardly discussed in literature. Only Shieh et al. ${ }^{6}$ propose a successive watermarking scheme where the first mark is embedded in a vector quantization domain whereas the second watermarking schemes operates in the middle DCT frequency band. Frequently, re-watermarking is only used as a special form of attack when watermark robustness should be investigated, no systematic investigations with respect to watermark interference as done in this work has been conducted previously. Most of the research effort has been invested into segmented watermarking, however, not a time or spatial domain segmentation is used (as proposed by Sheppard et al. ${ }^{5}$ and Woo et al. ${ }^{7}$ ) but instead the data partitioning is employed in a transform domain in most cases. Arnold et al. ${ }^{8}$ and Ganic et al. ${ }^{9,10}$ use the DFT and embed watermarks into different spectral bands, i.e. one into the low frequency band and a second one into the high frequency band. Wong et al. ${ }^{11}$ propose a scheme operating in the low frequency band of the DCT domain where the host coefficient data is disjoinlty partitioned among the watermarks to be inserted. Segmentation into low and high frequency wavelet subbands to embed two corresponding watermarks has been proposed by Lu et al. ${ }^{12,13}$ and Mehul et al., ${ }^{14}$ Tao et al. ${ }^{15}$ use four wavelet subbands for embedding.

With respect to the different intentions of multiple watermarking schemes as systematized by Mintzer et al., ${ }^{4}$ only the schemes proposed by Lu et al. ${ }^{13}$ and Woo et al. ${ }^{7}$ support multiple applications (i.e. copyright/annotation watermarks and authentication watermarks), all the other algorithms focus on multiple robust watermarking for ownership embedding. There are also differences concerning the purpose of multiple embedding of robust watermarks. Whereas the majority of the schemes described so far aims at embedding a single watermark multiple times in order to increase the robustness (the rationale behind this idea is to be resistant to a wider class of attacks if the mark is embedded in both high and low frequency bands), only few suggestions focus on embedding actually different watermarks. ${ }^{5,8,11}$

\section{MULTIPLE RE-WATERMARKING}

The embedding of unique watermarks for receiver identification is called fingerprinting. In case a cover medium is sold, it may be of interest that information concerning both, the original owner and the recipient, are embedded. In case re-selling occurs, each time the cover medium is sold the corresponding informations can be embedded using watermarking technology. In this case we can trace back the way of the cover medium to its origin and are able to reconstruct the entire trading chain. We want to support this scenario with multiple watermarking technology. Fingerprinting solves the question what to embed but not how to embed it.

Composite watermarking is not very useful in this scenario since the multiple watermarks are embedded simultanously. All watermarks to be embedded have to be present prior to embedding to generate the one single composite mark. Segmented watermarking suffers from the fact that at least the approximate number of watermarks to be embedded needs to be known in advance. Additionally, the techniques developed so far are restricted in terms of the number of marks that can be embedded. Therefore, successive or re-watermarking seems to be the most promising approach for our target scenario. Obviously, all watermarks embedded serve the same purpose so the multiple applications case does not apply.

Fig. 1 vizualizes our target scenario. We embed three marks $(A, B, C)$ successively into the image $I$ using some embedding technique $\oplus$. After inserting the marks, we result in the respectively marked images $I_{A}, I_{A, B}$, and $I_{A, B, C}$.

Sheppard et al. ${ }^{5}$ employ the blind spatial domain algorithm of Kalker et al. ${ }^{16}$ and the non-blind DCT domain algorithm of Cox et al. ${ }^{17}$ for multiple re-watermarking by simply embedding different marks successively. They report good performance in the first case and decreasing watermark correlations in the second case. In recent work $^{18}$ we have shown similar results for the non-blind wavelet algorithms by Wang and Corvi and the blind 


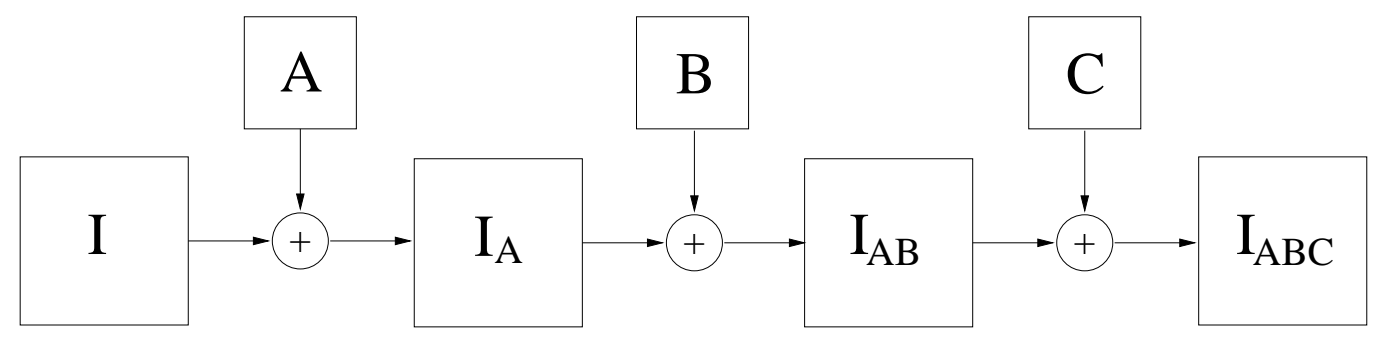

Figure 1. Multiple Re-Watermarking scenario.

DCT algorithm by Koch (see next section). Figs. 2 and 3 illustrate these findings in a setting as described in the subsequent section. The result graphs have to be read as follows: 10 watermarks have been embedded and the final image containing all marks is fed into the detection process. The mark at the rightmost position has been embedded as the last mark, all other indicated positions are ordered in time in the same manner (average, minimal and maximal correlation values of 50 experiments are given).

Fig. 2.a displays a typical behaviour for non-blind algorithms when used in multiple re-watermarking: whereas the last embedded watermark can be detected with a correlation value equal to that of single embedding, the detection correlation value decreases for watermarks further left in the plot (embedded at an earlier stage). The figure also shows that a surprisingly large number of marks can be detected reliably, provided the decision threshold is set accordingly.

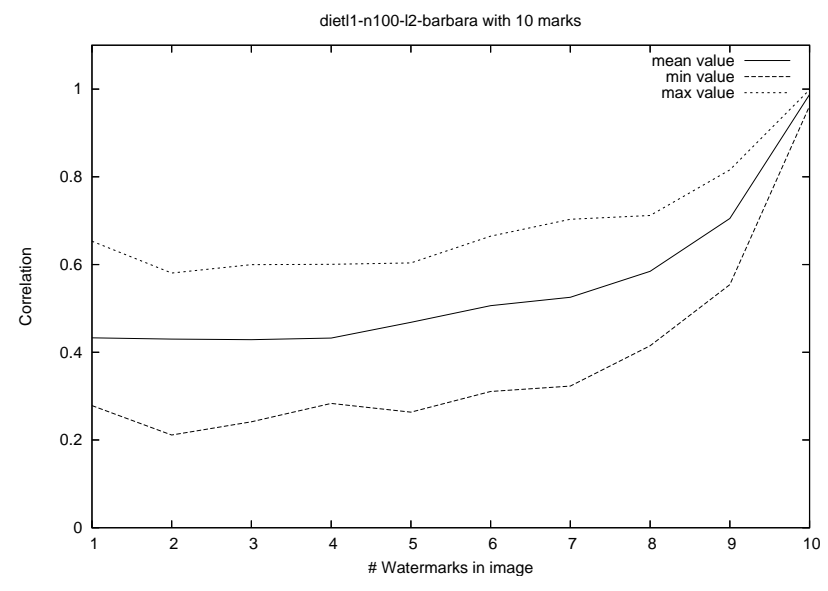

(a) Wang algorithm, $40 \mathrm{~dB}$

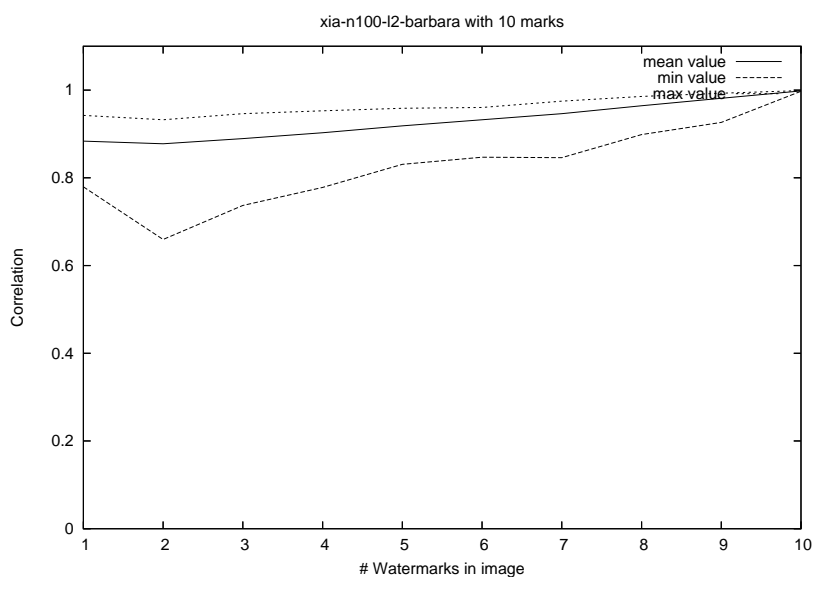

(b) Xia algorithm, $21 \mathrm{~dB}$

Figure 2. Detection response of non-blind algorithms for embedding 10 watermarks (50 experiments, results averaged), Barbara image.

The decrease in correlation is obviously due to watermark interference which is strongest for the first mark embedded (at the leftmost position) since the signal extracted for detection (the difference between the marked image containing all embedded marks $I_{A, B, C}$ and the original image $I$ in this case) is a signal involving all embedded watermarks. In the Wang algorithm, large transform coefficients are selected to be manipulated for watermark embedding - on the one hand the set of marked coefficients changes from one marking stage to the next (which causes the detection to lose synchronization), on the other hand a significant number of coefficients is selected repeatedly, which causes the watermark embedding process to partially overwrite the previously embedded information which degrades correlation.

Fig. 2.b shows that the correlation reduction can be limited: the Xia algorithm does not use an image 
dependent embedding pattern as does the Wang algorithms (which avoids the synchronization problem) and shows much better detection correlation, however, the embedding strength has been chosen rather high resulting in poor image quality. This shows, that the watermark interference problem has to be solved in a different manner.

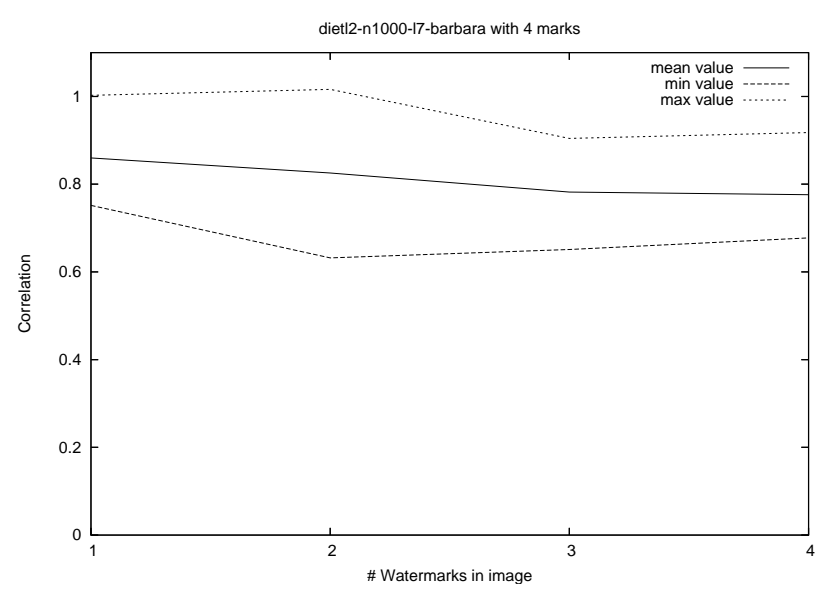

(a) 4 WM embedded

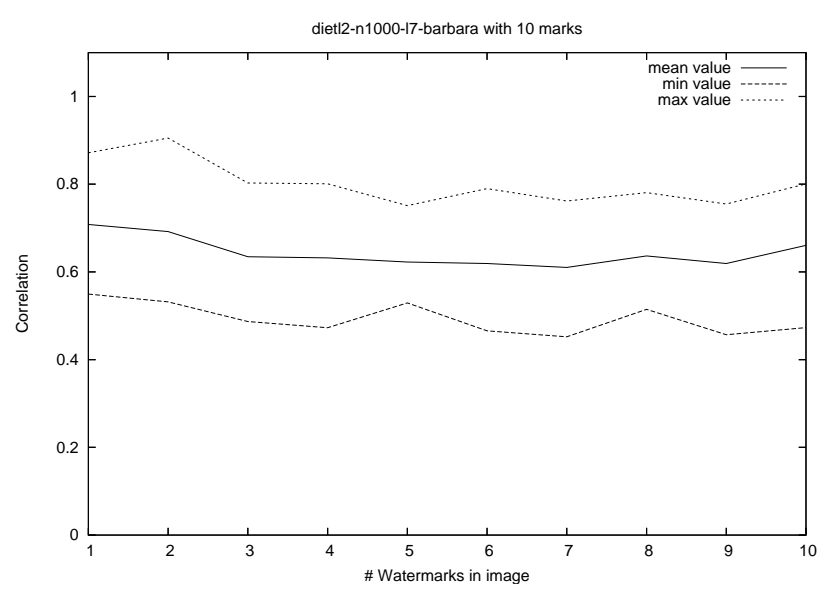

(b) 10 WM embedded

Figure 3. Detection response of the (blind) Dugad algorithm, ${ }^{19}$ Barbara image.

As displayed in Fig. 3, a similar phenomenon may be observed for blind algorithms. The detection correlation values decrease for an increasing number of embedded marks (they are already below those for single marking when four marks are embedded), but contrasting to the non-blind case they do not depend on the embedding order. The decrease in correlation is also due to watermark interference, but for blind techniques the signal used for detection is the original with all marks embedded $I_{A, B, C}$ which is correlated with the mark to be detected.

We want to investigate how the observed watermark interference can be avoided or decreased. The approach of Shieh et al. ${ }^{6}$ shows a possible strategy by using two entirely different embedding techniques, in particular different domains (i.e. a vector quantization and DCT domain, respectively) are used. Additionally, most segmented watermarking approaches embed watermarks in disjoint frequency bands hoping to increase robustness. In the following experiments we want to answer the question whether the use of different embedding domains or the use of different frequency bands helps to avoid watermark interference in re-watermarking and we will investigate which of the two approaches seems to be more profitable.

\section{EXPERIMENTAL STUDY}

\subsection{Setting and Methods}

For our experiments we have chosen a set of ten $8 \mathrm{bpp}$ and $512 \times 512$ pixels host images for watermark embedding, among them the well known images "Lena", "Barbara", and "Peppers" for which we provide detailed results. Furthermore, the images Crowd, Bridge, Lake, Lax, and images \#4, \#16, and \#41 from a collection of images available at http://decsai.ugr.es/cvg/CG/base.htm have been used. We have used the "Watermarking Toolbox" * developed by Peter Meerwald for watermark embedding and detection, unless denoted otherwise all embeddings have been done with the default parameters of the implementation using an embedding strength to result in an final host image PSNR of $38 \mathrm{~dB}$ after multiple watermark embedding in order to facilitate a fair comparison. Non-blind techniques are operated with correct reference images (images potentially already containing several marks before embedding the mark subject to detection, e.g. $I_{A}$ for $\operatorname{detecting}$ mark $B$ and $I_{A, B}$

\footnotetext{
${ }^{*}$ http://www.cosy.sbg.ac.at/ pmeerw/Watermarking/source/
} 
for detecting mark $C$ - instead of the original $I$ ) in the detection process. All experiments have been repeated 10 times using randomly chosen watermarks, averaged correlation results are presented. In these experiments, different watermarks and different embedding keys are chosen each time for embedding.

We have used a wide variety of algorithms for our experiments. The majority is wavelet-based (DWT): the principles of the algorithms by Xie, Corvi, Wang, Kim, Zhu, and Xia are described in our survey on waveletbased watermarking. ${ }^{19}$ Additionally we employ the global DCT-based algorithm by Cox, ${ }^{20}$ the block-based DCT algorithm by Koch, ${ }^{21}$ and a spatial algorithm by Bruyndonckx. ${ }^{22}$ The latter two algorithms operate on small image blocks and consequently they operate in the middle and high frequency band due to the limited spatial resolution. The remaining algorithms span a wide spectrum with respect to the frequency band the watermark embedding is performed. The Cox algorithm operates in the low frequency band, whereas for the wavelet based schemes the order given in the listing above corresponds to an increase of the frequency of the band the embedding takes place. The Kim algorithm is an exception since it embeds into both, low and high frequency bands, respectively.

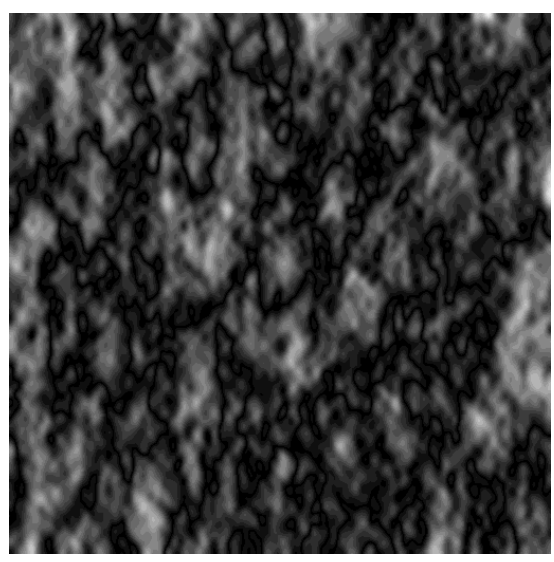

(a) Cox

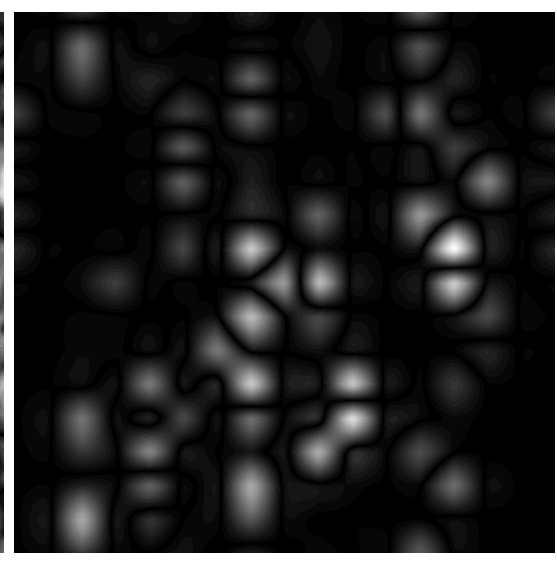

(b) Xie

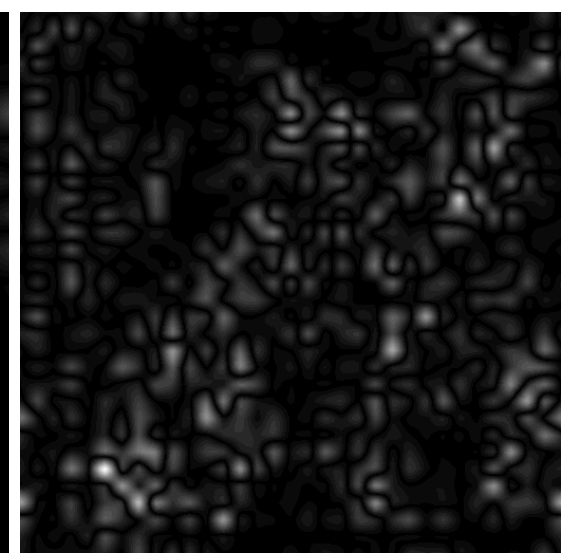

(c) Corvi

Figure 4. Difference image computed between original and watermarked version (low frequency watermarks).

The frequency band used for embedding is also visualized in Figs. 4 and 5 where we display the difference images between the original Lena image and an image with embedded watermark, which means that actually the embedded watermark information is visualized. The transition from low frequency information in Figs. 4.a-b to high frequency content (edges in this case) in Fig. 5.c is clearly visible.

The actual experiments are conducted as follows. We embed two watermarks $A, B$ with a single algorithm using re-watermarking in this order resulting in the image $I_{A, B}$ (compare Fig. 1) - this is the algorithm given in the captions of the following tables. The detection correlation of the first mark $A$ in $I_{A, B}$ is recorded and stated in brackets in each line after the name of the image used in all following tables. Subsequently, a third watermark $C$ is embedded into $I_{A, B}$ using a different algorithm resulting in $I_{A, B, C}$ - these algorithms constitute the columns in the tables. Finally, the detection correlation of the first watermark $A$ in $I_{A, B, C}$ is determined again and listed in the table (in the column of the algorithm that was used for embedding the third mark $C$ ). The rationale of this procedure is as follows: if the detection correlation of the mark $A$ in $I_{A, B, C}$ is significantly reduced as compared to the correlation value without third mark embedded (detection in $I_{A, B}$ ), mark $C$ obviously interferes strongly with mark $A$. The amount of interference can be seen by the amount of reduction of the detection correlation of mark $A$. Only the influence of several algorithms on the detection correlation of a single algorithm is studied (and values given in the tables), therefore we are not troubled by the question how to compare detection correlations of different algorithms. In addition to providing results for the abovementioned three images, we also show the averaged correlation values of all ten images in the tables. 


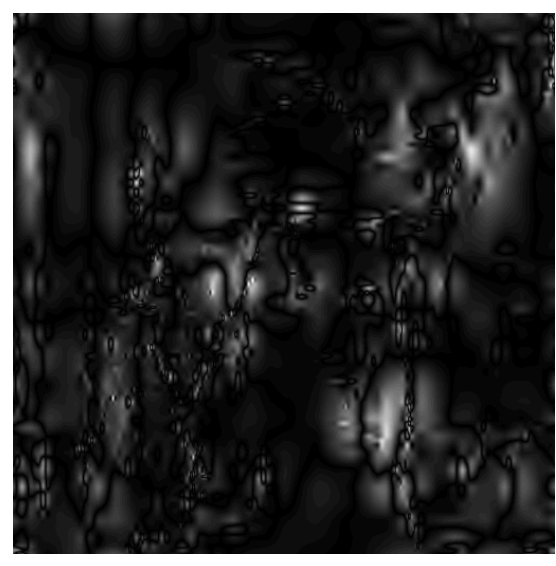

(a) Wang

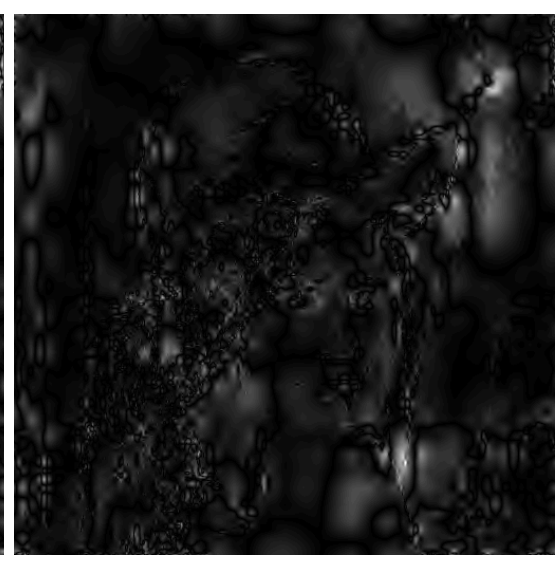

(b) Zhu

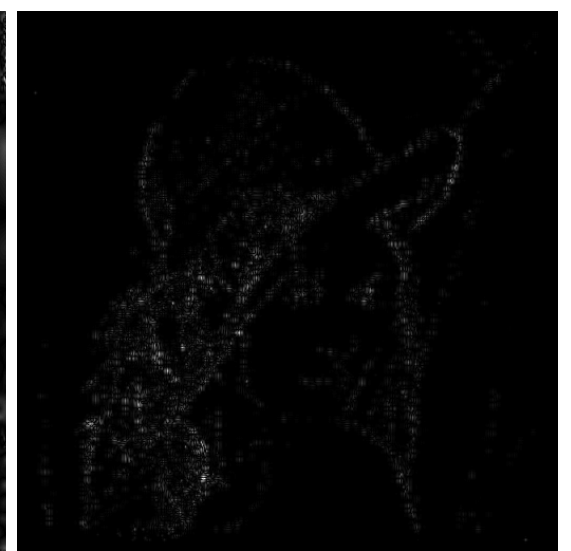

(c) Xia

Figure 5. Difference image computed between original and watermarked version (middle and high frequency watermarks).

\subsection{Results}

Table 1 displays the results in case the first two marks have been embedded using the Cox algorithm (which is a low frequency band DCT algorithm). It is evident that simply changing the embedding domain for embedding additional watermarks is not a good idea. Correlations in case of embedding the third mark using the Xie and Kim algorithms (both wavelet-based) are lowest. These algorithms embed into the low frequency band as does the Cox algorithm which obviously leads to significant watermark interference. The remaining algorithms considered all operate in the middle or high frequency band and no matter which embedding domain they use, detection correlation values close to the value with two embedded marks are delivered which indicates low interference.

\begin{tabular}{|l||c||c||c|c|c|c|c|c||}
\hline Image & Koch & Bruyn & Xie & Corvi & Wang & Kim & Zhu & Xia \\
\hline \hline Lena $(\mathbf{0 . 7 1})$ & 0.69 & 0.72 & 0.57 & 0.67 & 0.67 & 0.62 & 0.72 & 0.71 \\
\hline Barbara $(\mathbf{0 . 7 0})$ & 0.70 & 0.70 & 0.46 & 0.65 & 0.63 & 0.68 & 0.72 & 0.73 \\
\hline Peppers $(\mathbf{0 . 7 1})$ & 0.74 & 0.70 & 0.57 & 0.67 & 0.67 & 0.65 & 0.67 & 0.70 \\
\hline \hline Average $\mathbf{( 0 . 7 0 )}$ & 0.71 & 0.71 & 0.56 & 0.66 & 0.67 & 0.64 & 0.70 & 0.71 \\
\hline
\end{tabular}

Table 1. Cox: Correlations with embedded third mark.

Again, Table 2 shows that simply changing the embedding domain is not profitable. No matter which embedding domain is used, both low frequency embedding schemes (Cox-DCT and Xie-DWT) deliver significantly worse correlation results as compared to the case when high frequency embedding algorithms are used to embed the third mark, even though some of them (Zhu and Xia) embed in the DWT domain as does the Corvi algorithm.

\begin{tabular}{|l||c||c||c||c|c|c|c|c||}
\hline Image & Cox & Koch & Bruyn & Xie & Wang & Kim & Zhu & Xia \\
\hline \hline Lena $(\mathbf{0 . 4 5})$ & 0.29 & 0.49 & 0.45 & 0.33 & 0.36 & 0.34 & 0.46 & 0.46 \\
\hline Barbara $(\mathbf{0 . 4 6})$ & 0.30 & 0.46 & 0.46 & 0.33 & 0.35 & 0.36 & 0.46 & 0.47 \\
\hline Peppers $(\mathbf{0 . 4 6})$ & 0.32 & 0.48 & 0.48 & 0.33 & 0.36 & 0.37 & 0.47 & 0.47 \\
\hline \hline Average $(\mathbf{0 . 4 3})$ & 0.29 & 0.44 & 0.44 & 0.32 & 0.34 & 0.35 & 0.43 & 0.44 \\
\hline
\end{tabular}

Table 2. Corvi: Correlations with embedded third mark.

In Table 3 we observe a less pronounced interfeence behaviour for the low frequency band embedding DWT 
algorithm Xie as seen in the previous tables. The expected poor results of the other low frequency band embedding schemes (Cox, Corvi) do show up on the one hand, on the other hand correlation values are also reduced for the middle frequency embedding schemes Wang and Kim (where the interference seems to originate more from the identical embedding domain). Overall, the Xie algorithm is obviously very robust with respect to watermark interference in general, no significant correlation reduction is observed for any scheme.

\begin{tabular}{|l||c||c||c||c|c|c|c|c||}
\hline Image & Cox & Koch & Bruyn & Corvi & Wang & Kim & Zhu & Xia \\
\hline \hline Lena $(\mathbf{0 . 5 3})$ & 0.46 & 0.56 & 0.53 & 0.52 & 0.47 & 0.47 & 0.54 & 0.54 \\
\hline Barbara $(\mathbf{0 . 5 2})$ & 0.43 & 0.51 & 0.46 & 0.51 & 0.39 & 0.45 & 0.48 & 0.46 \\
\hline Peppers $(\mathbf{0 . 4 9})$ & 0.38 & 0.48 & 0.50 & 0.47 & 0.41 & 0.45 & 0.48 & 0.54 \\
\hline \hline Average $(\mathbf{0 . 5 1})$ & 0.40 & 0.51 & 0.52 & 0.44 & 0.41 & 0.44 & 0.50 & 0.52 \\
\hline
\end{tabular}

Table 3. Xie: Correlations with embedded third mark.

Table 4 shows the results when the DWT-based Kim algorithm is used for embedding the first two marks interestingly, detection correlation is not significantly reduced by subsequent low-frequency embedding schemes. This would have been expected since in Tables 1 and 2 it has been shown that the Kim algorithm reduces detection correlations of the low-frequency embedding Cox and Corvi schemes. Detection correlation is reduced most by the Zhu and Xia algorithms, embedding into the high frequency band. Recall that the Kim algorithm embeds into low frequency as well as into high frequency bands which is the reason for this interesting phenomenon. The DWT-based Kim algorithm is the only case where we observe an important role of the domain used for embedding - only techniques embedding into the DWT domain as well exhibit reduced correlation values (but only in a specific band).

\begin{tabular}{|l||c||c||c||c|c|c|c|c||}
\hline Image & Cox & Koch & Bruyn & Xie & Corvi & Wang & Zhu & Xia \\
\hline \hline Lena $(\mathbf{0 . 7 1})$ & 0.69 & 0.66 & 0.70 & 0.62 & 0.69 & 0.60 & 0.37 & 0.54 \\
\hline Barbara $\mathbf{( 0 . 7 2 )}$ & 0.72 & 0.65 & 0.71 & 0.69 & 0.70 & 0.68 & 0.31 & 0.36 \\
\hline Peppers $\mathbf{( 0 . 7 2 )}$ & 0.68 & 0.66 & 0.69 & 0.65 & 0.70 & 0.61 & 0.35 & 0.40 \\
\hline \hline Average $\mathbf{( 0 . 7 4 )}$ & 0.72 & 0.68 & 0.72 & 0.69 & 0.72 & 0.67 & 0.35 & 0.40 \\
\hline
\end{tabular}

Table 4. Kim: Correlations with embedded third mark.

Finally, Table 5 displays the results of the high frequency band embedding DWT algorithm Xia. In general, the correlation values found are very high indicating a good suitability for multiple embedding applications of this algorithm in general. However, the only reduction observed is found in the case of the high frequency band embedding schemes Koch and Zhu, where the DWT scheme Zhu (identical embedding domain) exhibits the most significant correlation decrease.

\begin{tabular}{|l||c||c||c||c|c|c|c|c||}
\hline Image & Cox & Koch & Bruyn & Xie & Corvi & Wang & Kim & Zhu \\
\hline \hline Lena $(\mathbf{0 . 9 2})$ & 0.91 & 0.88 & 0.91 & 0.91 & 0.91 & 0.90 & 0.91 & 0.82 \\
\hline Barbara $(\mathbf{0 . 9 7})$ & 0.97 & 0.96 & 0.97 & 0.97 & 0.97 & 0.97 & 0.97 & 0.94 \\
\hline Peppers $(\mathbf{0 . 8 7})$ & 0.87 & 0.83 & 0.86 & 0.87 & 0.87 & 0.86 & 0.86 & 0.72 \\
\hline \hline Average $(\mathbf{0 . 9 6})$ & 0.96 & 0.94 & 0.95 & 0.96 & 0.96 & 0.96 & 0.95 & 0.91 \\
\hline
\end{tabular}

Table 5. Xia: Correlations with embedded third mark.

\section{CONCLUSIONS AND FUTURE WORK}

It has been demonstrated that watermark interference in multiple re-watermarking applications can be limited better by using disjoint frequency bands for embedding the different marks as compared to simply using different 
embedding domains. Only in case of the watermarks are all embedded into the same frequency band for some reason, it is preferable to use different embedding domains. In future work we will investigate the use of parameterized transform domains to tackle the watermark interference problem in re-watermarking scenarios.

\section{Acknowledgements}

The work described in this paper is partially supported by the Austrain Science Fund, project no. 15170 and has mostly been done in the context of a multimedia lab at CTI.

\section{REFERENCES}

1. I. J. Cox, M. L. Miller, and J. A. Bloom, Digital Watermarking, Morgan Kaufmann, 2002.

2. S. Katzenbeisser and F. A. P. Petitcolas, Information Hiding Techniques for Steganography and Digital Watermarking, Artech House, Dec. 1999.

3. B. Furht and D. Kirovski, eds., Multimedia Security Handbook, CRC Press, Boca Raton, Florida, 2005.

4. F. Mintzer and G. W. Braudaway, "If one watermark is good, are more better?," in Proceedings of the International Conference on Accoustics, Speech, and Signal Processing, 4, pp. 2067-2070, (Phoenix, Arizona, USA), May. 1999.

5. N. P. Sheppard, R. Shafavi-Naini, and P. Ogunbona, "On multiple watermarking," in Proceedings of the ACM Multimedia and Security Workshop 2001 (MMSW-01), pp. 3-6, ACM Press, (Ottawa, Canada), Oct. 2001.

6. C.-S. Shieh, H.-C. Huang, F.-H. Wang, and J.-S. Pan, "An embedding algorithm for multiple watermarks," Journal of Information Science and Engineering 19, pp. 381-395, Mar. 2003.

7. C. S. Woo, J. Du, and B. Pham, "Multiple watermark method for privacy control and tamper detection in medical images," in APRS Workshop on Digital Image Computing (WDIC 2005), B. C. Lovell and M. A. J, eds., pp. 43-48, The Australian Pattern Recognition Society (APRS), The University of Queensland, (St. Lucia, QLD 4072 Australia), Feb. 2005.

8. M. Arnold and Z. Huang, "Blind detection of multiple audio watermarks," in Proceedings of First International Conference on Web Delivering of Music, 2001, P. Nesi, P. Bellini, and C. Busch, eds., pp. 4-11, IEEE Computer Society, (Florence, Italy), Nov. 2001.

9. E. Ganic and A. M. Eskicioglu, "A DFT-based semi-blind multiple watermarking scheme for images," in 4th New York Metro Area Networking (NYMAN 2004) Workshop, (The Graduate Center of the City University of New York), Sep. 2004.

10. E. Ganic, D. Scott, and A. M. Eskicioglu, "Embedding multiple watermarks in the DFT, domain using low and high frequency bands," in Proceedings of SPIE: Security, Steganography, and Watermarking of Multimedia Contents VII, E. J. Delp III and P. W. Wong, eds., 5681, pp. 175-184, (San Jose, CA, USA), Mar. 2005.

11. P. H. W. Wong and O. C. Au, "A blind watermarking technique for multiple watermarks," in Proceedings of the IEEE 2003 International Symposium on Circuits and Systems, 2, pp. 936-939, (Bangkok, Thailand), May. 2003.

12. C. S. Lu, S.-K. Huang, C. J. Sze, and H. Y. M. Liao, "Cocktail watermarking for digital image protection," IEEE Transactions on Multimedia 2, pp. 209-224, Dec. 2000.

13. C.-S. Lu and H.-Y. M. Liao, "Multipurpose watermarking for image authentication and protection," in Proceedings of IEEE Transaction on Image Processing, 10, pp. 1579-1592, IEEE Signal Processing Society, Oct. 2001.

14. R. Mehul and R. Priti, "Discrete wavelet transform based multiple watermarking scheme," in IEEE Region 10 Technical Conference on Convergent Technologies for the Asia-Pacific (TENCON), 3, pp. 935-938, (Bangalore), Oct. 2003.

15. P. Tao and A. M. Eskicioglu, "A robust multiple watermarking scheme in the discrete wavelet transform domain," in Proceedings of SPIE: Internet Multimedia Management Systems V, J. R. Smith, T. Zhang, and S. Panchanathan, eds., 5601, pp. 133-144, Oct. 2004. 
16. T. Kalker, G. Depovere, J. Haitsma, and M. Maes, "A video watermarking system for broadcast monitoring," in Proc. SPIE Security and Watermarking of Multimedia Contents, 3657, pp. 103-112, SPIE, (San Jose, CA), Jan. 1999.

17. I. J. Cox, J. Kilian, T. Leighton, and T. Shamoon, "A secure, robust watermarking for multimedia," in Proceedings of the First International Workshop on Information Hiding, Lecture Notes in Computer Science 1174, pp. 185-206, Springer-Verlag, (London, UK), 1996.

18. A. Mascher-Kampfer, H. Stögner, and A. Uhl, "Multiple re-watermarking scenarios," in $13^{\text {th }}$ International Conference on Systems, Signals, and Image Processing (IWSSIP'06), (Budapest, Hungary), 2006. To appear.

19. P. Meerwald and A. Uhl, "A survey of wavelet-domain watermarking algorithms," in Proceedings of SPIE, Electronic Imaging, Security and Watermarking of Multimedia Contents III, P. W. Wong and E. J. Delp, eds., 4314, SPIE, (San Jose, CA, USA), Jan. 2001.

20. I. J. Cox, J. Kilian, T. Leighton, and T. G. Shamoon, "A secure, robust watermark for multimedia," in Information hiding: first international workshop, R. Anderson, ed., Lecture Notes in Computer Science 1174, pp. 183-206, Springer Verlag, Berlin, Germany, (Cambridge, UK), 1996.

21. E. Koch and J. Zhao, "Towards robust and hidden image copyright labeling," in Proceedings of the IEEE International Workshop on Nonlinear Signal and Image Processing, pp. 452-455, (Marmaras, Greece), June 1995.

22. O. Bruyndonckx, J.-J. Quisquater, and B. M. Macq, "Spatial method for copyright labeling of digital images," in Proceedings of the IEEE International Workshop on Nonlinear Signal and Image Processing, pp. 456-459, (Marmaras, Greece), 1995. 